\title{
Biomimetic synthesis of poly(lactic-co-glycolic acid)/multi-walled carbon nanotubes/apatite composite membranes
}

\author{
H. L. Zhang* \\ College of Stomatology, Ningxia Medical University, 750004 Yinchuan, China
}

Received 23 November 2011; accepted in revised form 15 February 2012

\begin{abstract}
Bioactive guided tissue regeneration (GTR) membrane has had some success for periodontal therapy. In this study, poly(lactic-co-glycolic acid) (PLGA)/multi-walled carbon nanotubes (MWNTs) composite membranes were incubated in three supersaturated calcification solutions (SCS) of different $\mathrm{pH}$ values for 21 days to prepare a PLGA/ MWNTs/apatite composite. Scanning electron microscope (SEM), X-ray diffraction (XRD), Fourier transform infrared spectroscopy (FTIR), energy dispersive spectroscopy (EDS), water contact angle measurement and mechanical testing were used for characterization. It was found that after 21 days incubation, apatite with low crystallite size and crystallinity was formed on the PLGA/MWNTs composite membranes. The Ca-poor carbapatite was similar in morphology and composition to that of natural bone. The size and shape of the apatite crystals immersed in three SCS were different from each other. The hydrophilicity and mechanical properties of the PLGA/MWNTs composite membranes were significantly enhanced after mineralization. This indicated that biomimetic mineralization may be an effective method to improve the biocompatibility and bone inductivity of certain materials. The PLGA/MWNTs/apatite composites may be potentially useful in GTR applications, particularly as GTR membranes for periodontal tissue regeneration.
\end{abstract}

Keywords: polymer composites, biomineralization, apatite crystal, GTR membrane

\section{Introduction}

Periodontitis is a common oral disease, which is the main reason for tooth loss in the adult world population. The ultimate goal of periodontal therapy is to restore the lost structure and function of the periodontal tissue, including the regeneration of periodontal membrane, cementum and alveolar bone. Traditional periodontal treatment can only control inflammation, but it is difficult to achieve the effect of regeneration. Therefore, how to regenerate the lost periodontal tissue has already become a key issue in periodontal therapy. However, the technique of guided tissue regeneration (GTR), proposed by Nyman et al. [1] in the early 1980s, has had some success. In this treatment technology, GTR membrane is the key factor that directly affects the final restoration results.

An ideal GTR membrane should be biocompatible and biodegrade to nontoxic products within a specific time scale $[2,3]$. It should be easy to fabricate with proper mechanical properties [4, 5] and present a penetrating structure $[6,7]$ that plays a role of blocking as well as transporting the nutrients and metabolic waste of the tissue $[8,9]$. However, the GTR membranes currently in clinical use still have many problems, such as mechanical strength, degradation rate and the host immune response. Researchers apply a variety of new materials and new technologies to this area, trying to develop the membranes which meet the GTR requirements.

\footnotetext{
${ }^{*}$ Corresponding author, e-mail: hua31415926@163.com

(c) BME-PT
} 
From the perspective of materials science, single material and single structure fail to meet the above requirements, therefore, the ideal GTR membrane should have multi-layer composite structure.

Poly(lactic-co-glycolic acid) (PLGA) and multiwalled carbon nanotubes (MWNTs) are two materials commonly used in tissue engineering. PLGA has attracted significant attention for its application in soft tissue engineering, bone tissue engineering, drug delivery, and nerve regeneration, due to its biocompatibility and biodegradability [10]. MWNTs have good biocompatibility when in contact with blood, bone, cartilage and soft tissue. Moreover, MWNTs have excellent biomedical properties, and can dramatically increase the mechanical properties of the composites $[11,12]$. Therefore, they may both serve as candidates for GTR applications. However, PLGA has poor hydrophilicity for osteoprogenitor cellar vitality and specific cell interactions. Hydroxyapatite (HA), a major inorganic component of natural bone, on the other hand, exhibits good biocompatibility and osteoconductivity, which is generally suggested for effective acceleration of new bone formation $[13,14]$. Therefore, HA coatings have been widely used in order to improve the biocompatibility and bone inductivity of the hydrophobic materials [15].

Mineralized composite membranes or scaffolds have been reported by many researchers by using different mineralization methods. Yang et al. [16] has reported mineralization of chitosan/MWNTs composite membranes by alternate soaking process and found that orientated nanoscopic crystallites of apatite were formed on the surface of the composites. However, this method needed to replace mineralization solutions every $12 \mathrm{~h}$ and the $\mathrm{Ca} / \mathrm{P}$ molar ratio was $\sim 2.6$ to 3.0 , which was rather a lot higher than that of stoichiometric hydroxyaptite (1.67). Corncob/HA scaffold has been fabricated by simulated body fluid (SBF) method by Ye et al. [17], but preparation of the mineralization solutions was somewhat complicated and time consuming. Supersaturated calcification solutions (SCS) method is an easy and effective way for preparing novel and bioactive composite materials [18]. Moreover, mineralization of PLGA/MWNTs composite membranes by SCS method has hardly been reported.

In this study, we are aiming at synthesizing the PLGA/MWNTs/apatite composites by biomimetic mineralization, studying the formation of apatite on PLGA/MWNTs membranes, and evaluating various properties before and after mineralization, as a foundation for further study of the PLGA/MWNTs/ apatite composites for GTR application.

\section{Experimental}

\subsection{Materials}

PLGA $\left(M_{\mathrm{w}}=100000 \mathrm{~g} / \mathrm{mol}\right)$ with a lactide/glycolide ratio of 75:25 was purchased from Shandong Daigang Co. Ltd. (Jinan, China). MWNTs (diameter: $8-15 \mathrm{~nm}$, length: $0.5 \mu \mathrm{m}$, purity $>95 \mathrm{wt} \%$ ) were purchased from Chengdu Organic Chemistry Co. Ltd. (Chengdu, China). Dimethyl formamide (DMF), trichloromethane (TCM) and other chemical reagents (A.R.) were purchased from Tianjin Chemical Reagent Co., Ltd. (Tianjin, China).

\subsection{Preparation of PLGA/MWNTs composite membranes}

PLGA/MWNTs composite membranes were prepared using the solution casting technique. Briefly, PLGA (2 g) was dissolved in a $20 \mathrm{~mL}$ mixture of TCM and DMF (volume ratio 7:3). MWNTs (0.1 g) were added to the PLGA solution and the mixture was then ultrasonicated for $1 \mathrm{~h}$ to disperse the MWNTs. The mixture was poured into a glass culture dish (diameter $12 \mathrm{~cm}$ ) and placed in the ventilating cabinet for $48 \mathrm{~h}$ to evaporate the solvent, resulting in a membrane $\sim 0.1 \mathrm{~mm}$ thick. All experiments were carried out in air and the ambient condition was $25^{\circ} \mathrm{C}$ and $60 \%$ humidity.

\subsection{Mineralization of the composite membranes}

In this study, three supersaturated calcification solutions (SCS) of different $\mathrm{pH}$ values (SCS1, SCS2 and SCS3) were selected for mineralization [18]. The ion concentrations and $\mathrm{pH}$ values of the three supersaturated calcification solutions are shown in Table 1. The composite membranes $(22 \mathrm{~mm} \times$ $22 \mathrm{~mm}$ ) were soaked in a freshly prepared saturated $\mathrm{Ca}(\mathrm{OH})_{2}$ solution at room temperature for one hour first, and rinsed with distilled water. Then the membranes were divided into three groups, immersed in three different SCS respectively, and incubated at $37^{\circ} \mathrm{C}$ for 21 days to determine the mineralization behavior in vitro. The three SCS were replaced every $24 \mathrm{~h}$. At the end of the incubation time, the samples 
Table 1. The ion concentrations $[\mathrm{mM} / \mathrm{L}]$ and $\mathrm{pH}$ values of the three supersaturated calcification solutions

\begin{tabular}{|l|c|c|c|c|c|c|}
\hline & $\mathbf{N a}^{+}$ & $\mathbf{C a}^{2+}$ & $\mathbf{C l}^{-}$ & $\mathbf{H}_{2} \mathbf{P O}_{4}{ }^{-}$ & $\mathbf{H C O}_{3}{ }^{-}$ & $\mathbf{p H}$ \\
\hline SCS1 & 6.5 & 5 & 10 & 5 & 1.5 & 5.96 \\
\hline SCS2 & 4 & 5 & 10 & 2.5 & 1.5 & 6.31 \\
\hline SCS3 & 4 & 10 & 20 & 2.5 & 1.5 & 6.20 \\
\hline
\end{tabular}

were rinsed with deionized water and then dried under vacuum at room temperature.

\subsection{Characterization of composite membranes}

The morphology of the samples was observed with SEM (JSM-7500F, JEOL, Japan). FTIR (Nicolet 560 , Nicolet Co., USA) was used in the range from 4000 to $400 \mathrm{~cm}^{-1}$ for the analysis of the crystals formed on the surface of the composites. Elemental analysis of the crystals was by EDS (JSM-7500F, JEOL, Japan). An X-ray diffractometer (SA-HF3, Rigaku, Japan) was used to investigate the mineral crystals grown on the surface of the membranes. The XRD was carried out with a Ni-filtered CuKa radiation source operated at a voltage of $40 \mathrm{kV}$ and a current of $30 \mathrm{~mA}$. The samples were scanned from 3 to $80^{\circ}(2 \theta)$ and the scan rate was $8^{\circ} / \mathrm{min}$. The wettability of the samples was determined by water contact angle measurement using JGW-360B (Midwest group, Beijing, China). Samples were placed on a sample stage and a single drop $(20 \mu \mathrm{L})$ of distilled water was dropped at three different places and the contact angle was then measured. The mechanical properties of the samples were analyzed using a universal testing machine (UTM, Instron 5567, USA) at room temperature. The extension rate was kept at $5 \mathrm{~mm} / \mathrm{min}$ and the load cell used was $100 \mathrm{~N}$ with a gauge length of $25 \mathrm{~mm}$. The dimensions of the samples were $10 \mathrm{~mm} \times 70 \mathrm{~mm}$ $(W \times L)[19]$.

\section{Results and discussion}

\subsection{Crystalline structure by XRD}

The X-ray diffractogram of PLGA/MWNTs composite membranes, and the samples immersed in three different SCS for 21 days are shown in Figure 1. The XRD observations revealed that the PLGA/ MWNTs composites were amorphous. The inorganic phase in the mineralized samples was determined as apatite crystals from the presence of several characteristic XRD diffraction peaks. It can

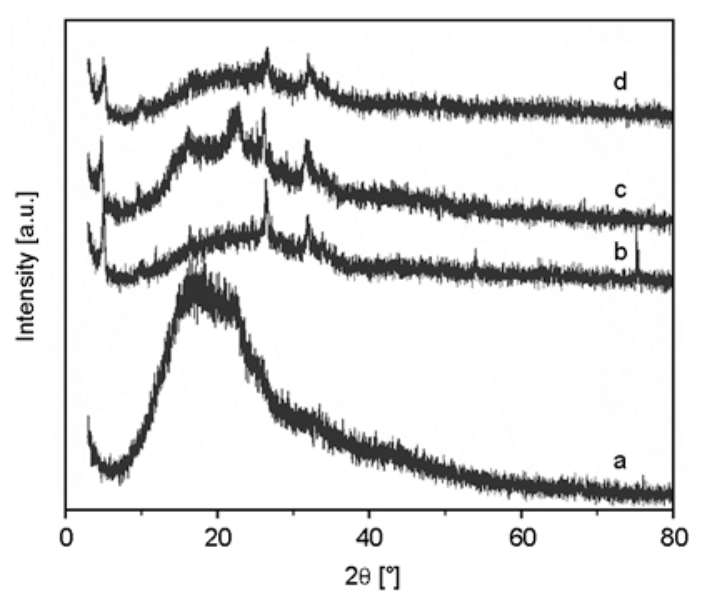

Figure 1. XRD patterns of (a) PLGA/MWNTs composite membranes, and PLGA/MWNTs composite membranes after immersion in (b) SCS1, (c) SCS2 and (d) SCS3 for 21 days

be seen that the samples immersed in SCS1, SCS2, and SCS3 all showed main diffraction peaks at $2 \theta=$ 26 and $32^{\circ}$, which were corresponded to planes 002 and 211 of HA. The samples immersed in SCS1 also showed the other peak at $2 \theta=54^{\circ}$, which was caused by the plane 004 of HA. However, the other diffraction peaks of HA were not found. Besides, the peaks of 211, 112 and 300 overlapped with each other without division, suggesting that the crystallinity of the apatite crystals was very low. Compared with JCPDS standard card of HA, the peaks of the apatite crystals in the mineralized samples became wider, which indicated that the crystallite size was tiny and the crystallinity was very low, closely resembling that of the natural bone tissue $[20,21]$. Moreover, the diffraction peak at $2 \theta=26^{\circ}$ of apatite in group SCS1 was the highest and sharpest, followed by that in group SCS2 and then group SCS3, which may indicate that the sizes of the apatite crystals in three groups are in the same order.

Another notable feature was that the peak at $2 \theta=$ $26^{\circ}(002)$ was higher and sharper than that at $2 \theta=$ $32^{\circ}(211)$, indicating that the mineral crystals preferred orientation growth along the (002) surface of the C-axis of HA. HA is a hexagonal columnar crystal, and $\mathrm{OH}$ groups arranges in the $\mathrm{C}$-axis or (002) plane. (002) direction is the preferred growth direction. The atoms accumulated along the (002) plane densely, which caused the formation of needle-like or lamellar appearance of the crystals. The mineral crystals grew along a certain axis, making 
the overlap of some planes or defects of crystal growth, which also contributed to the lack of the other diffraction peaks of HA [22]. As C-axis of HA in natural bone tissue aligned along the collagen fibers, it has a certain significance to study how the organic matrix template controls the growth of apatite in biomimetic mineralization.

\subsection{FTIR analysis of the mineral crystals and PLGA/MWNTs composite membranes}

The apatite crystals were scraped off the surface of the PLGA/MWNTs composite membranes immersed in SCS2 for analysis by FTIR. The FTIR spectrum of the PLGA/MWNTs composite membranes was also presented for comparison. As shown in Figure 2, the FTIR spectrum of the apatite crystals showed characteristic $\mathrm{PO}_{4}{ }^{3-}$ absorption bands at 1036,603 , and $566 \mathrm{~cm}^{-1}$ and $\mathrm{O}-\mathrm{H}$ absorption band

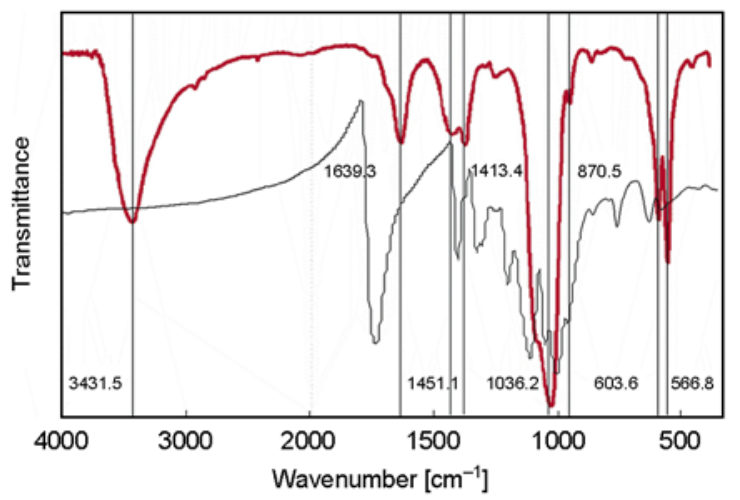

Figure 2. FTIR spectrum of the PLGA/MWNTs composite membranes (black) and the apatite crystals scraped off the surface of the PLGA/MWNTs composite membranes immersed in SCS2 for 21 days (red)

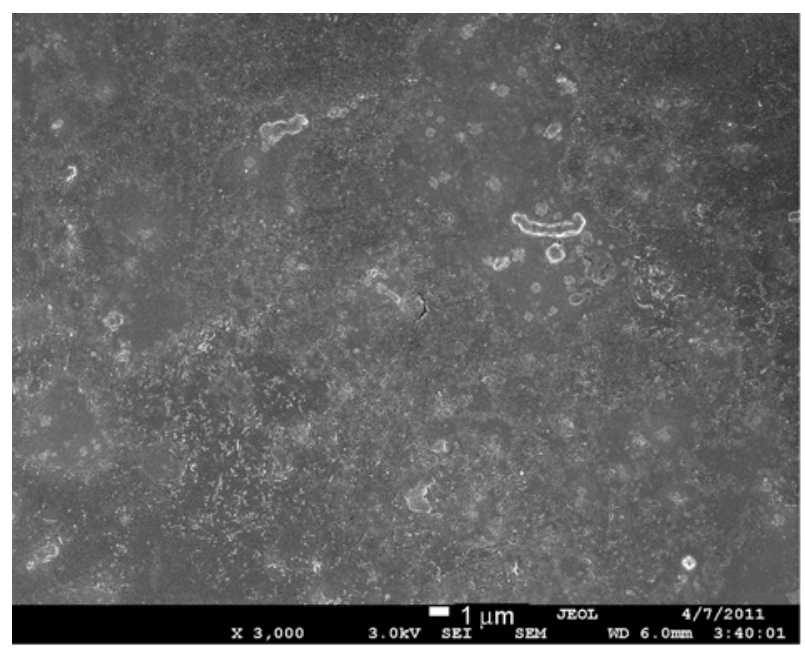

a) around $3431 \mathrm{~cm}^{-1}$. The difference of the $\mathrm{PO}_{4}{ }^{3-}$ absorption bands between the apatite crystals grown on the surface of the composites and HA was that the absorption peak of the former around 1000 $\sim 1100 \mathrm{~cm}^{-1}$ was not split, indicating a lower crystallinity of apatite coating. The $\mathrm{CO}_{3}{ }^{2-}$ absorption bands appeared at 1451,1413 , and $870 \mathrm{~cm}^{-1}$, indicating that $\mathrm{CO}_{3}{ }^{2-}$ entered the crystal lattice structure of apatite. The apatite coating was composed of carbapatite, which was similar to the composition of HA in natural bone tissue.

\subsection{Surface morphology by SEM}

The SEM images of uncoated PLGA/MWNTs composite membranes are shown in Figure 3. It can be seen that the structure of the uncoated PLGA/ MWNTs composite membranes was relatively wellproportioned and identical. There was no bubble or hole on the surface of the membranes. The SEM image taken at a higher magnification in Figure $3 \mathrm{~b}$ reveals that MWNTs which were short tubular shaped were well dispersed and embedded in PLGA matrix, indicating that good alignment of MWNTs was achieved in PLGA matrix during the preparation of the composite membranes.

After 21 days of mineralization, the surfaces of the three samples were all covered by a layer of white mineralized crystals, but the mineral solutions still remained clear, indicating that heterogeneous nucleation occurred on the surfaces of the composite membranes $[23,24]$. As shown in SEM images presented in Figure 4, the surfaces of all the PLGA/ MWNTs composite membranes showed an obvious

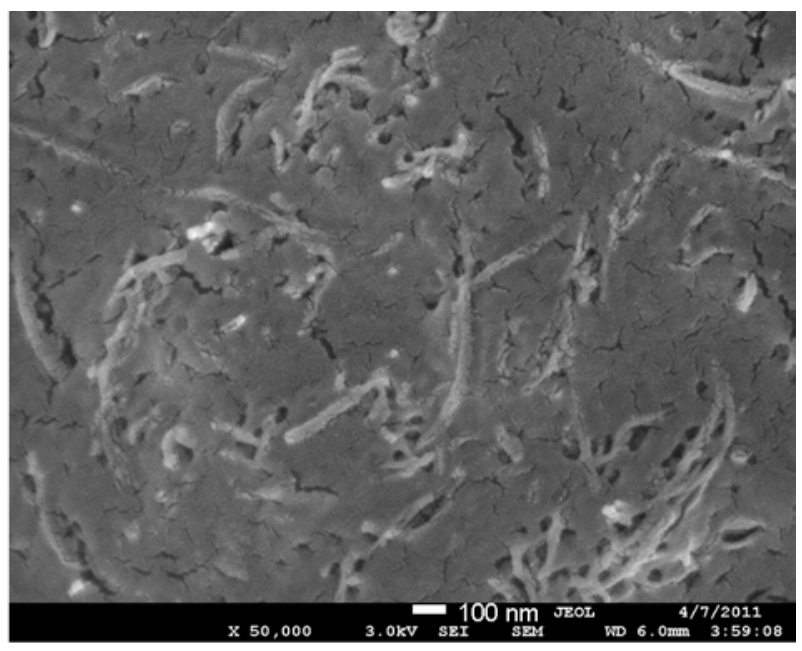

b)

Figure 3. SEM images of PLGA/MWNTs composite membranes (a) at a magnification of 3000; (b) at a magnification of 50000 

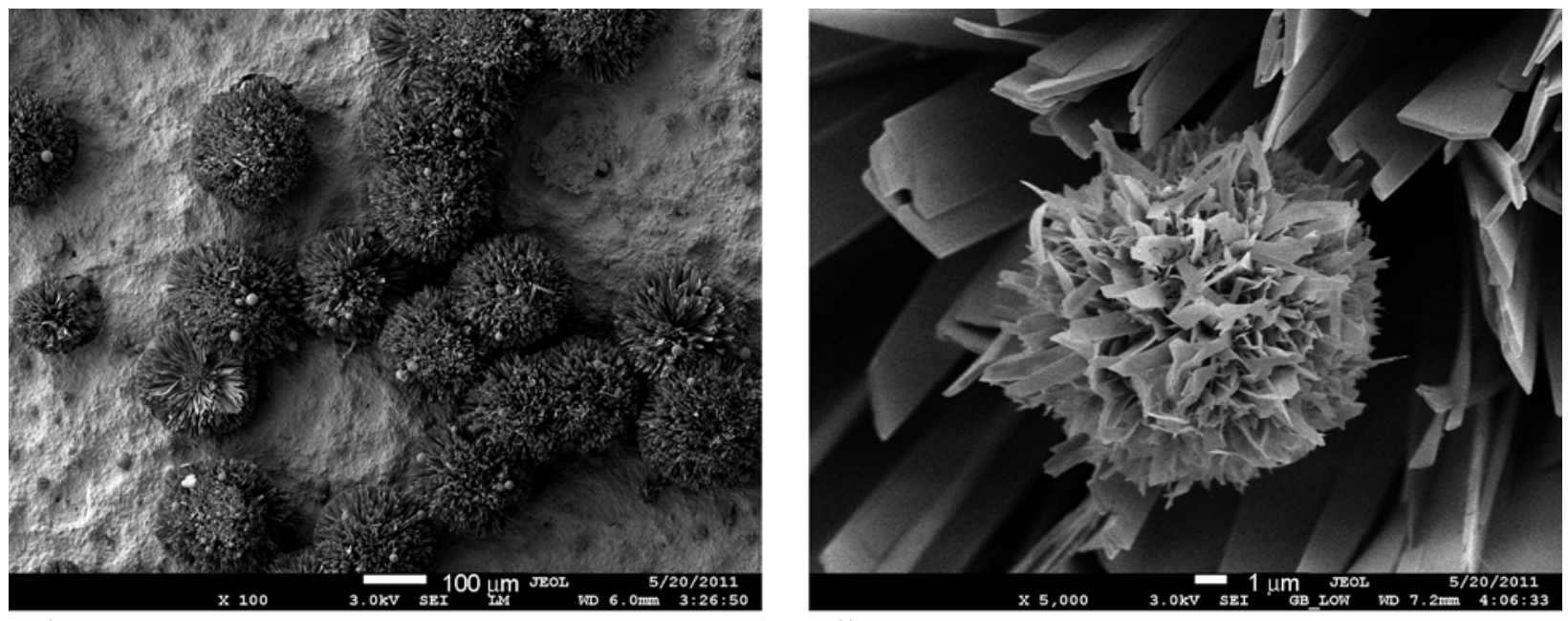

a)
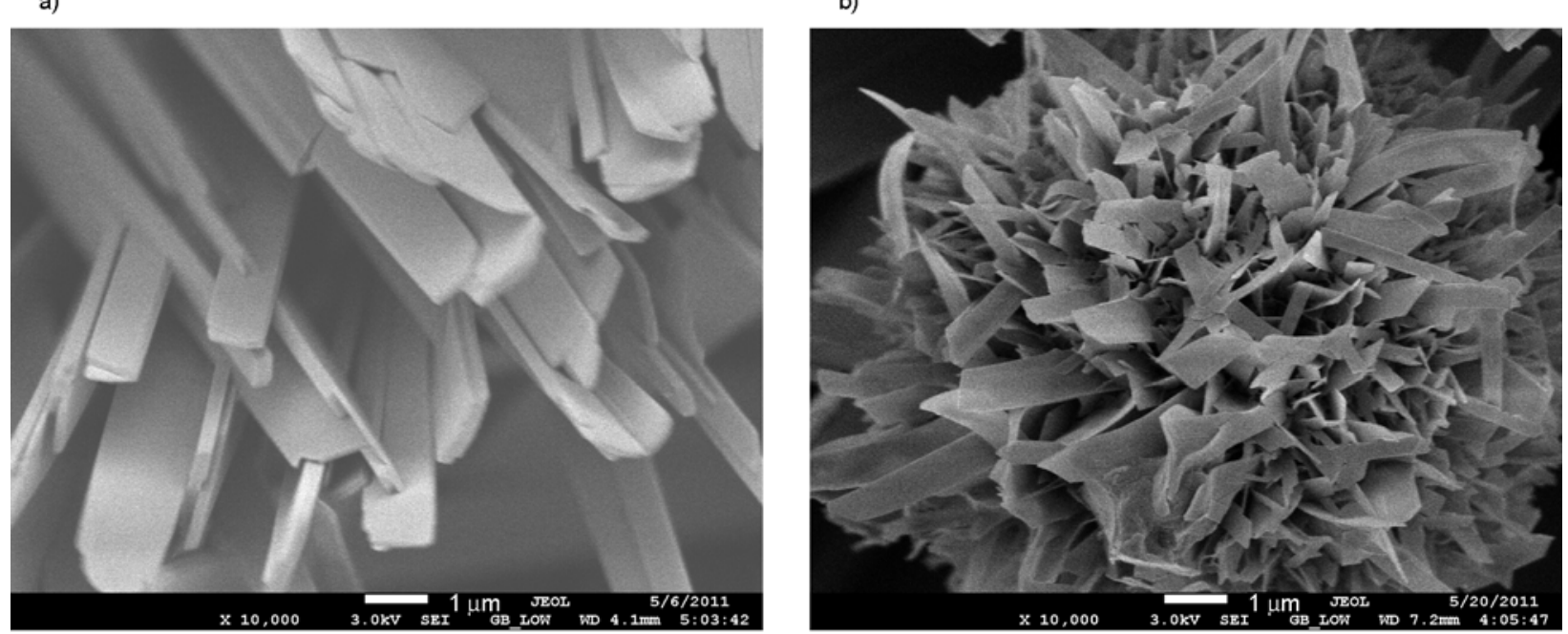

c)

d)

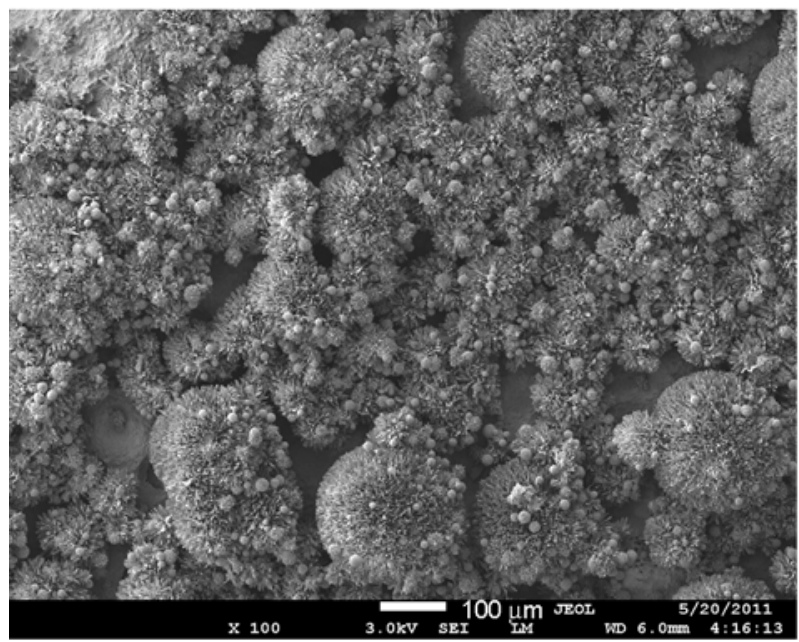

e)

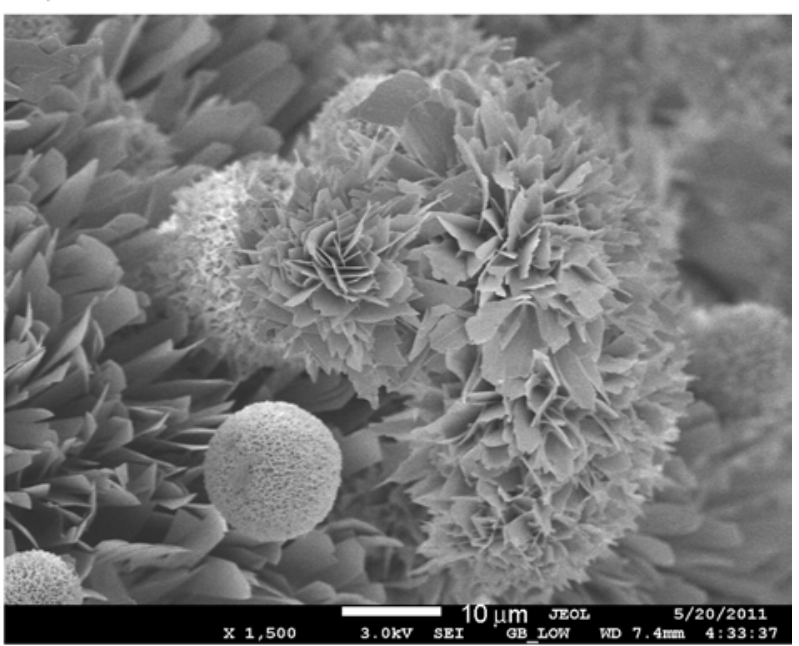

f)

Figure 4. SEM images of PLGA/MWNTs composite membranes immersed in (a) (b) (c) (d) SCS1, (e) (f) (g) (h) SCS2, and (i) (j) (k) (l) SCS3 for 21 days (continued on next page)

change after mineralization. The apatite crystals clustered together regularly to form spherical particles on the surface of the composite membranes. However, the morphology of the crystals was slightly different from each other due to the different ion concentrations and $\mathrm{pH}$ values of SCS. The SEM images taken at a higher magnification reveal that the flat quadrilateral-shaped single crystals at 


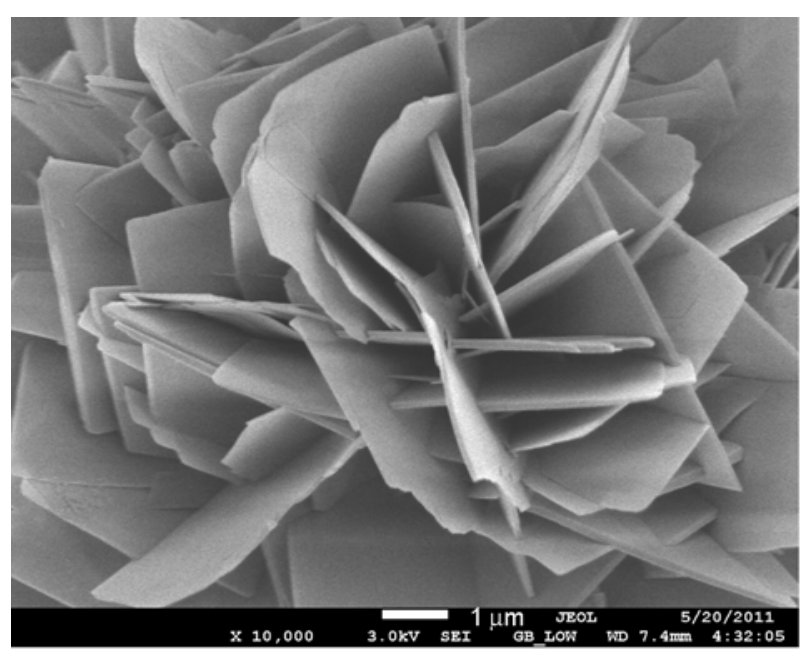

g)

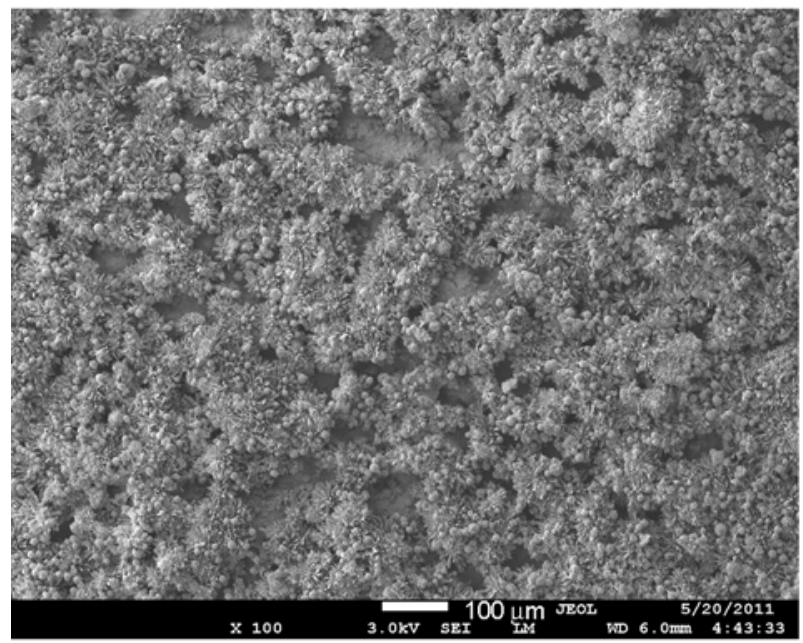

i)

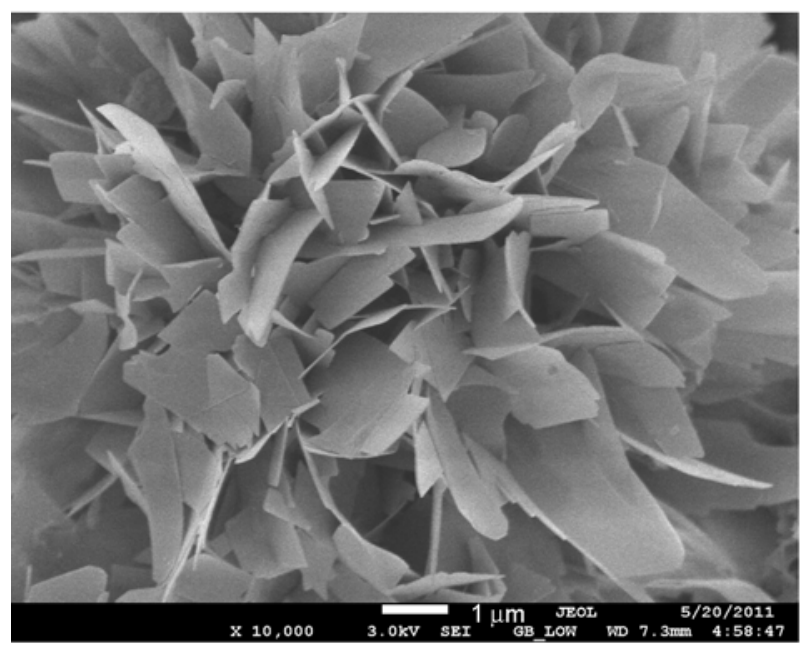

k)

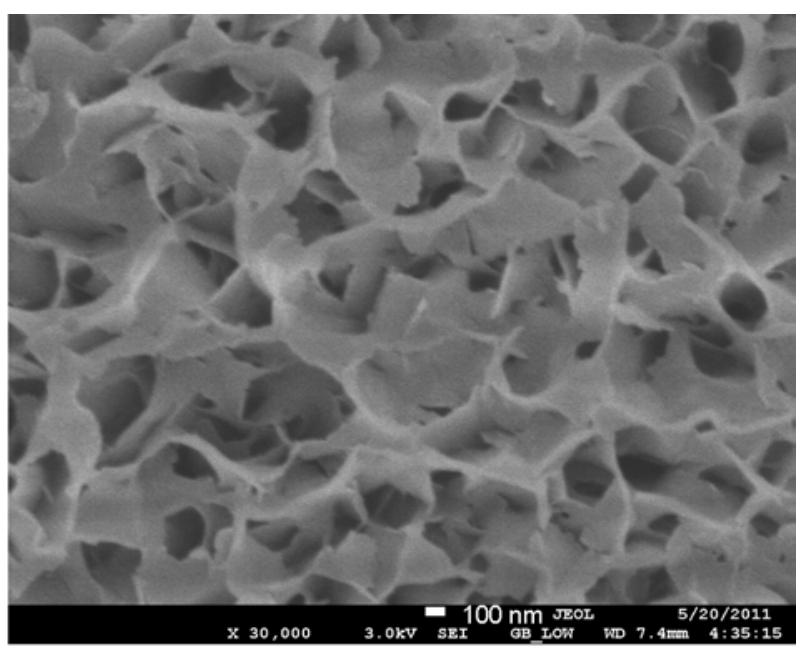

h)

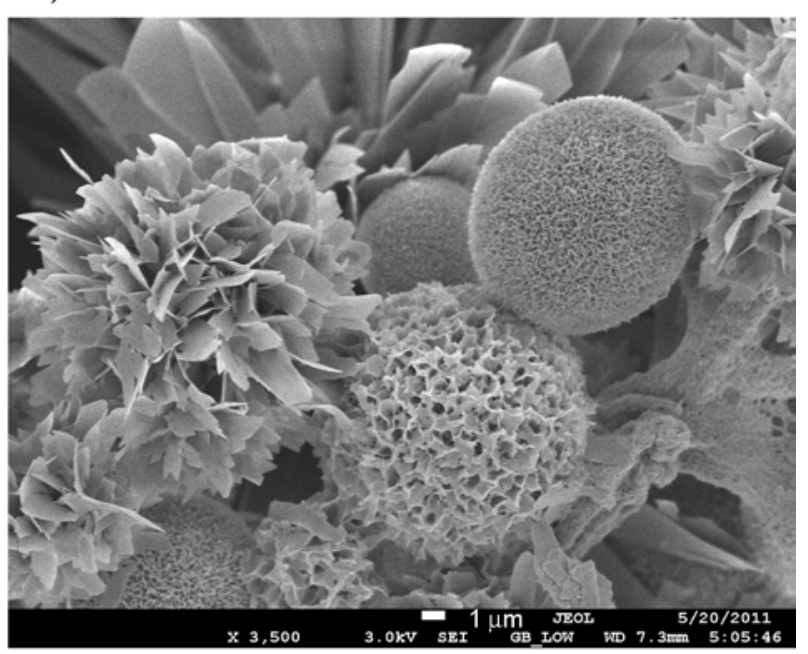

j)

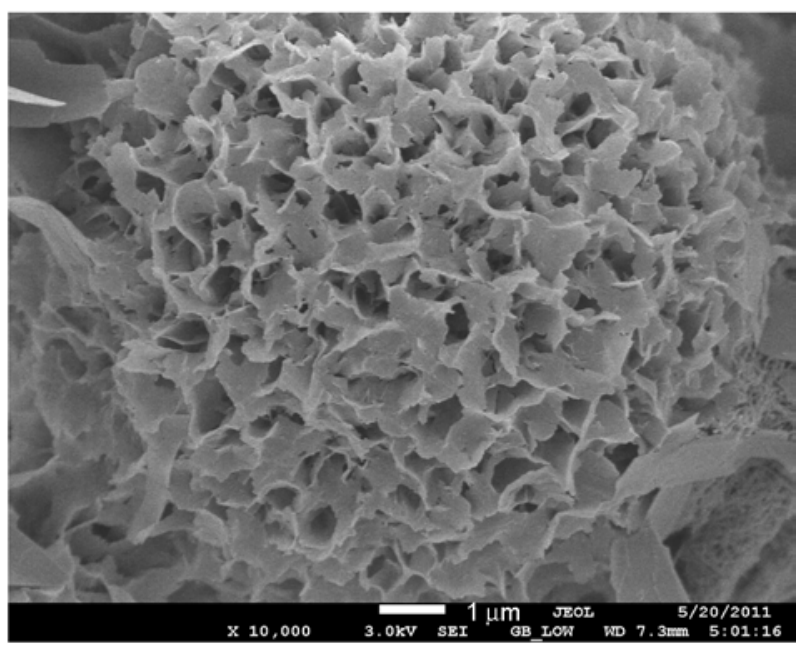

l)

Figure 4. (continued form the previous page) SEM images of PLGA/MWNTs composite membranes immersed in (a) (b) (c) (d) SCS1, (e) (f) (g) (h) SCS2, and (i) (j) (k) (l) SCS3 for 21 days

the bottom layer, which formed from the original calcium and phosphorus deposits, interwove into a bundle. These crystals formed flowers-like shape and covered the entire surface of the matrix. Above these crystals, it can be seen that tiny and slender quadrilateral-shaped crystals were formed in group SCS1, and leaf-shaped and honeycomb-shaped crystals were formed uniformly in group SCS2 and 
SCS3, indicating that the crystals previously formed served as secondary nucleation sites for additional mineral formation. Besides, the amount of the apatite crystals in group SCS3 was the most, followed by that in group SCS2 and then group SCS1. Moreover, the size of the apatite crystals was the largest in group SCS1, followed by that in group SCS2 and then group SCS3, which also confirmed the results of XRD patterns. As for how the ion concentrations and $\mathrm{pH}$ values affect the size and shape of the crystals, further study is needed.

\subsection{Elements analysis}

EDS was used to analyze the type and proportion of elements of crystals. As shown in Figure 5, the evident presence of $\mathrm{Ca}$ and $\mathrm{P}$ peaks indicated the presence of HA on all the surfaces of the three samples. Table 2 shows the $\mathrm{Ca} / \mathrm{P}$ molar ratios determined
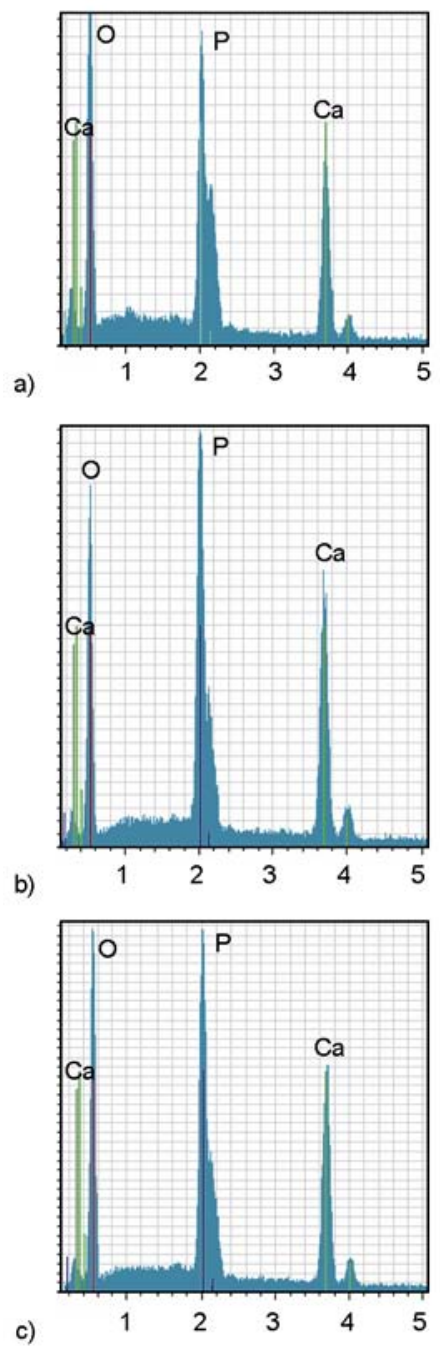

Figure 5. EDS scan results from the crystals of PLGA/ MWNTs composite membranes immersed in (a) SCS1, (b) SCS2 and (c) SCS3 for 21 days
Table 2. $\mathrm{Ca} / \mathrm{p}$ ratio of apatite on the surface of different samples

\begin{tabular}{|c|c|}
\hline & $\mathbf{C a} / \mathbf{P}$ \\
\hline SCS1 & 1.59 \\
\hline SCS2 & 1.43 \\
\hline SCS3 & 1.46 \\
\hline
\end{tabular}

from EDS analysis. $\mathrm{Ca} / \mathrm{P}$ molar ratios of the samples immersed in SCS1, SCS2 and SCS3 were 1.59, 1.43 and 1.46 , respectively, which were all slightly lower than that of the chemical dose-HA $(\mathrm{Ca} / \mathrm{P}=$ 1.67). Since the crystals contain carbonate ion substituted at phosphate ion lattice sites, therefore, the coatings are calcium-deficient-carbonated apatite and the apatite deposited on the surface of the composite membranes is Ca-poor apatite [25].

\subsection{Wettability analysis}

In this study, hydrophilicity of the surface of PLGA, PLGA/MWNTs, and the PLGA/MWNTs/ apatite membranes immersed in SCS2 for 21 days was measured by water contact angle (Table 3 ). The contact angle of pure PLGA membrane was $117.5 \pm 3.6^{\circ}$, which clearly demonstrated the hydrophobic nature of PLGA surface. Addition of MWNTs into PLGA matrix did not change the hydrophobic nature of PLGA/MWNTs surface and the contact angle $\left(114.7 \pm 3.1^{\circ}\right)$ was similar as that of the PLGA membrane. However, after mineralization, the surface of the PLGA/MWNTs/apatite membranes was completely hydrophilic (water contact angle $0^{\circ}$ ). Similar phenomenon was also observed by Lee et al. [26].

Table 3. Water contact angles of PLGA, PLGA/MWNTs and PLGA/MWNTs/apatite membranes immersed in SCS2 for 21 days

\begin{tabular}{|l|c|}
\hline \multicolumn{1}{|c|}{ Sample name } & Water contact angle $\left[{ }^{\circ}\right]$ \\
\hline PLGA & $117.5 \pm 3.6$ \\
\hline PLGA/MWNTs & $114.7 \pm 3.1$ \\
\hline PLGA/MWNTs/apatite & 0 \\
\hline
\end{tabular}

\subsection{Mechanical characterization}

Mechanical properties of PLGA, PLGA/MWNTs and PLGA/MWNTs/apatite membranes immersed in SCS2 for 21 days were shown in Table 4. Compared to the pure PLGA membrane, the mechanical properties of the PLGA/MWNTs membranes increased dramatically. The Young's modulus and 
Table 4. Young's modulus, tensile stress and elongation at break of three membranes

\begin{tabular}{|l|c|c|c|}
\hline \multicolumn{1}{|c|}{ Sample name } & Young's modulus [MPa] & Tensile stress [MPa] & Elongation at break [\%] \\
\hline PLGA & $152.0 \pm 16.6$ & $5.43 \pm 0.62$ & $36.8 \pm 4.69$ \\
\hline PLGA/MWNTs & $314.9 \pm 30.2$ & $6.03 \pm 1.03$ & $51.6 \pm 6.71$ \\
\hline PLGA/MWNTs/apatite & $382.4 \pm 34.4$ & $7.73 \pm 0.95$ & $11.2 \pm 2.17$ \\
\hline
\end{tabular}

tensile strength increased sharply by 107 and by $11 \%$, respectively. The elongation at break was increased by $40 \%$. It is evident that even a small amount of MWNTs would significantly improve the mechanical properties of the composites [17]. After mineralization, the Young's modulus and tensile strength of the PLGA/MWNTs/apatite membranes increased to $382.4 \pm 34.4$ and $7.73 \pm 0.95 \mathrm{MPa}$, respectively. This may be due to increase in rigidity over the PLGA/ MWNTs membranes after mineralization. However, the elongation at break decreased after mineralization, indicating that the PLGA/ MWNTs/apatite composites became somewhat stiffer compared to PLGA/MWNTs membranes. The results of mechanical test indicated that the mechanical properties of the PLGA/MWNTs/apatite composites and PLGA/MWNTs composites were greatly improved compared to the pure PLGA membrane.

\section{Conclusions}

Apatite crystals were coated on the surface of the PLGA/MWNTs composite membranes after immersion in SCS for 21 days, suggesting that the PLGA/ MWNTs composites have good biomineralization performance in vitro and it is feasible to prepare biomimetic materials for GTR by this method. The formed apatite with low crystallite size and crystallinity was Ca-poor carbapatite, which was similar in composition and structure to that of natural bone. The size and shape of the apatite crystals immersed in three SCS of different ion concentrations and $\mathrm{pH}$ values were different from each other, indicating that we may control the size and shape of the crystals through regulating the composition of the mineral solution. The PLGA/MWNTs membranes showed improved hydrophilicity and mechanical strength after mineralization. These novel PLGA/MWNTs/apatite composites with controlled macroscale and microscale configuration are expected to be a promising bioactive GTR membrane for periodontal tissue regeneration.

\section{Acknowledgements}

This work was supported by the Key Project of Ministry of Education of China (grant number: 211196), Natural Science Foundation of NingXia, China (grant number: NZ11124) and the School Program of Ningxia Medical University (grant number: XT201006).

\section{References}

[1] Nyman S., Lindhe J., Karring T., Rylander H.: New attachment following surgical treatment of human periodontal disease. Journal of Clinical Periodontology, 9, 290-296 (1982). DOI: 10.1111/j.1600-051X.1982.tb02095.x

[2] Brekke J. H., Toth J. M.: Principles of tissue engineering applied to programmable osteogenesis. Journal of Biomedical Materials Research, 43, 380-398 (1998). DOI: 10.1002/(SICI)1097-4636(199824)43:4<380::

\section{AID-JBM6>3.0.CO;2-D}

[3] Gunatillake P. A., Adhikari R.: Biodegradable synthetic polymer for tissue engineering. European Cells and Materials, 5, 1-16 (2003).

[4] Lin A. S. P., Barrows T. H., Cartmell S. H., Guldberg R. E.: Microarchitectural and mechanical characterization of oriented porous polymer scaffolds. Biomaterials, 24, 481-489 (2003). DOI: $10.1016 / \mathrm{S} 0142-9612(02) 00361-7$

[5] Kokubo T., Kim H-M., Kawashita M.: Novel bioactive materials with different mechanical properties. Biomaterials, 24, 2161-2175 (2003). DOI: $10.1016 / \mathrm{S} 0142-9612(03) 00044-9$

[6] Li S., de Wijn J. R., Li J. P., Layrolle P., de Groot K.: Macroporous biphasic calcium phosphate scaffold with high permeability/porosity ratio. Tissue Engineering, 9, 535-548 (2003). DOI: $10.1089 / 107632703322066714$

[7] Deschamps A. A., Claase M. B., Sleijster W. J., de Bruijn J. D., Grijpma D. W., Feijen J.: Design of segmented poly(ether ester) materials and structures for the tissue engineering of bone. Journal of Controlled Release, 78, 175-186 (2002). DOI: 10.1016/S0168-3659(01)00497-7

[8] Chen Y., Mak A. F. T., Wang M., Li J., Wong M. S.: PLLA scaffolds with biomimetic apatite coating and biomimetic apatite/collagen composite coating to enhance osteoblast-like cells attachment and activity. Surface and Coatings Technology, 201, 575-580 (2006). DOI: $10.1016 /$ j.surfcoat.2005.12.005 
[9] Goldstein A. S., Zhu G., Morris G. E., Meszlenyi R. K., Mikos A. G.: Effect of osteoblastic culture conditions on the structure of poly(DL-lactic-co-glycolic acid) foam scaffolds. Tissue Engineering, 5, 421-433 (1999).

DOI: $10.1089 /$ ten.1999.5.421

[10] Zhang H. L., Chen Z. Q.: Fabrication and characterization of electrospun PLGA/MWNTs/ hydroxyapatite biocomposite scaffolds for bone tissue engineering. Journal of Bioactive and Compatible Polymers, 25, 241-259 (2010). DOI: $10.1177 / 0883911509359486$

[11] Cao X., Dong H., Li C. M., Lucia L. A.: The enhanced mechanical properties of a covalently bound chitosanmultiwalled carbon nanotube nanocomposite. Journal of Applied Polymer Science, 113, 466-472 (2009).

DOI: 10.1002/app.29984

[12] Curtin W. A., Heldon B. W.: CNT-reinforced ceramics and metals. Materials Today, 11, 44-49 (2004). DOI: 10.1016/S1369-7021(04)00508-5

[13] Sheikh F. A., Kanjwal M. A., Macossay J., Barakat N. A. M., Kim H. Y.: A simple approach for synthesis, characterization and bioactivity of bovine bones to fabricate the polyurethane nanofiber containing hydroxyapatite nanoparticles. Express Polymer Letters, 6, 41-53 (2012).

DOI: $10.3144 /$ expresspolymlett.2012.5

[14] Tan Q., Zhang K., Gu S., Ren J.: Mineralization of surfactant functionalized multi-walled carbon nanotubes (MWNTs) to prepare hydroxyapatite/MWNTs nanohybrid. Applied Surface Science, 255, 7036-7039 (2009). DOI: 10.1016/j.apsusc.2009.03.036

[15] Fathi M. H., Azam F.: Novel hydroxyapatite/tantalum surface coating for metallic dental implant. Materials Letters, 61, 1238-1241 (2007). DOI: 10.1016/j.matlet.2006.07.013

[16] Yang J., Yao Z., Tang C., Darvell B. W., Zhang H., Pan L., Liu J., Chen Z.: Growth of apatite on chitosan-multiwall carbon nanotube composite membranes. Applied Surface Science, 255, 8551-8555 (2009).

DOI: $10.1016 /$ j.apsusc. 2009.06.013

[17] Ye Y-M., Huang C-P., Wang Q., Li Q-L., Chen Z-Q., Bao C-Y.: Biomimetic synthesis of a novel HA/corncob composite. Applied Surface Science, 255, 548551 (2008).

DOI: 10.1016/j.apsusc.2008.06.056
[18] Li F., Feng Q. L., Cui F. Z., Li H. D., Schubert H.: A simple biomimetic method for calcium phosphate coating. Surface and Coatings Technology, 154, 88-93 (2002).

DOI: $10.1016 / \mathrm{S} 0257-8972(01) 01710-8$

[19] Zhang H.: Electrospun poly (lactic-co-glycolic acid)/ multiwalled carbon nanotubes composite scaffolds for guided bone tissue regeneration. Journal of Bioactive and Compatible Polymers, 26, 347-362 (2011). DOI: $10.1177 / 0883911511413450$

[20] Varma H. K., Yokogawa Y., Espinosa F. F., Kawamoto Y., Nishizawa K., Nagata F., Kameyama T.: Porous calcium phosphate coating over phosphorylated chitosan film by a biomimetic method. Biomaterials, 20, 879-884 (1999).

DOI: $10.1016 / \mathrm{S} 0142-9612(98) 00243-9$

[21] Yang C. R., Wang Y. J., Chen X. F., Zhao N. R.: Biomimetic fabrication of $\mathrm{BCP} / \mathrm{COL} / \mathrm{HCA}$ scaffolds for bone tissue engineering. Materials Letters, 59, 36353640 (2005).

DOI: $10.1016 /$ j.matlet.2005.07.022

[22] Varma H. K., Yokogawa Y., Espinosa F. F., Kawamoto Y., Nishizawa K., Nagata F., Kameyama T.: In-vitro calcium phosphate growth over functionalized cotton fibers. Journal of Materials Science: Materials in Medicine, 10, 395-400 (1999).

DOI: 10.1023/A:1008970913107

[23] Jakobsen R. J., Brown L. L., Hutson T. B., Fink D. J., Veis A.: Intermolecular interactions in collagen selfassembly as revealed by Fourier transform infrared spectroscopy. Science, 220, 1288-1290 (1983).

DOI: $10.1126 /$ science. 6857249

[24] Linde A.: Dentin mineralization and the role of odontoblasts in calcium transport. Connective Tissue Research, 33, 163-170 (1995). DOI: $\underline{10.3109 / 03008209509016997}$

[25] Li L., Li G., Wang Y., Jiang J.: Apatite formation on poly (vinyl alcohol)-coated poly ( $\varepsilon$-caprolactone) films by incubation in simulated body fluids. Applied Surface Science, 255, 7734-7738 (2009). DOI: $10.1016 /$ j.apsusc.2009.04.154

[26] Lee J. H., Rim N. G., Jung H. S., Shin H.: Control of osteogenic differentiation and mineralization of human mesenchymal stem cells on composite nanofibers containing poly[lactic-co-(glycolic acid)] and hydroxyapatite. Macromolecular Bioscience, 10, 173182 (2010).

DOI: $\underline{10.1002 / \mathrm{mabi} .200900169}$ 